\title{
Médiévales
}

Langues, Textes, Histoire

$64 \mid$ printemps 2013

Temporalités de l'Égypte

\section{Ferdinando RAFFAELE (éd.), Lu raxunamentu di l'abbati Moises e di lu beatu Germanu supra la virtuti di la discretioni}

Palerme, Centro di studi filologici e linguistici siciliani, 2009, 204 p. (« Supplementi al Bollettino », 17)

Jérémy Delmulle

\section{(2) OpenEdition}

Journals

Édition électronique

URL : https://journals.openedition.org/medievales/7026

DOI : 10.4000/medievales.7026

ISSN : $1777-5892$

Éditeur

Presses universitaires de Vincennes

Édition imprimée

Date de publication : 1 juillet 2013

Pagination : 188-190

ISBN : 978-2-84292-371-6

ISSN : 0751-2708

Référence électronique

Jérémy Delmulle, «Ferdinando raffaele (éd.), Lu raxunamentu di l'abbati Moises e di lu beatu Germanu supra la virtuti di la discretioni », Médiévales [En ligne], 64 I printemps 2013, mis en ligne le 09 septembre 2013, consulté le 24 avril 2022. URL : http://journals.openedition.org/medievales/7026 ; DOI : https:// doi.org/10.4000/medievales.7026

Ce document a été généré automatiquement le 24 avril 2022.

Tous droits réservés 


\title{
Ferdinando RAFFAELE (éd.), $L u$
} raxunamentu di l'abbati Moises e di lu beatu Germanu supra la virtuti di la discretioni

Palerme, Centro di studi filologici e linguistici siciliani, 2009, 204 p. (« Supplementi al Bollettino », 17)

\author{
Jérémy Delmulle
}

1 La nouvelle publication dont Ferdinando Raffaele vient enrichir les travaux déjà nombreux du Centro di studi filologici e linguistici siciliani (qui s'est donné pour objectif d'éditer tous les textes de la littérature en langue sicilienne de la fin du MoyenÂge) est la version remaniée de sa thèse de doctorat, soutenue à l'Università di Catania en 1998. Elle consiste en l'édition critique d'un des derniers textes composés en vulgaire sicilien, qui est le volgarizzamento de la deuxième des Conférences de Jean Cassien, consacrée à la vertu de discrétion, édition à laquelle l'éditeur a ajouté une riche introduction et un très précieux glossaire, qui permettent tous deux de mieux mesurer à la fois la place du Raxunamentu dans la littérature en vulgaire sicilien et l'intérêt que présente ce témoin d'un point de vue linguistique.

2 Élaboré par un traducteur anonyme du monastère bénédictin de San Martino delle Scale, ce texte tardif - il est datable de la première moitié du xvi ${ }^{e}$ siècle (sans doute 1510-1550 ; cf. p. 128) - nous est connu par un unique manuscrit (S), copie de l'original (p.112), et constitue le cas rare et très intéressant d'une transmission séparée d'une seule Conférence (la conception de la Conférence comme œuvre à part entière va même jusqu'à la suppression, à l'intérieur du texte, des renvois internes à une autre partie du recueil [p. 49]) qui, par son sujet, la discretio, mère de toutes les vertus, et par la copie simultanée, dans le même volume, de la Regula Benedicti, trahit dès l'abord la destination monastique qu'on lui prévoit. Mais cette œuvre se rattache à une vaste tradition, surtout prospère aux XIV $\mathrm{XV}^{\mathrm{e}} \mathrm{XV}$ siècles; car les Conlationes de Cassien avaient donné lieu déjà à d'autres volgarizzamenti. 
3 Le Raxunamentu - mot qui traduit conlatio (conférence), le terme collationi étant réservé à une autre acception du mot, désignant le repas (trois occurrences ; cf. le glossaire, s.u., p. 145) - est donc un volgarizzamento entendu au sens (défini p. 42, n. 179) de "genre littéraire [consistant en] la transposition linguistique d'un texte d'un idiome qui est considéré du point de vue littéraire comme supérieur à un autre qui jouit d'un moindre prestige ». Mais il est surtout l'un des derniers témoins de cette littérature en vulgaire sicilien, puisqu'il voit le jour à une époque où cette langue a perdu tout usage communicationnel et n'est plus guère qu'un medium poétique que la toscanisation du vulgaire écrit est déjà parvenue à reléguer définitivement au second plan au profit d'une langue considérée comme une langue de culture.

4 L'introduction est précisément pour l'éditeur l'occasion de passer en revue l'ensemble de la littérature en vulgaire sicilien, afin de déterminer le contexte de la rédaction du Raxunamentu et de caractériser, même sans les identifier, le ou les auteurs ainsi que les lecteurs visés. Née à la cour de Frédéric III dans les années 1310, la littérature en vulgaire sicilien s'est rapidement caractérisée comme une complète réécriture d'œuvres latines (l'Énéide, les Faits et dits de Valère-Maxime, les Dialogues de Grégoire), plus que comme leur simple transposition (p.18-19). Précisément, l'analyse serrée du texte et la confrontation avec son modèle fait bien apparaître un vulgarisateur très à l'aise avec le latin pourtant difficile de Cassien : il a bien traduit la Conlatio et, sans passer par un volgarizzamento antérieur (p.43-46), les erreurs de compréhension sont quasi inexistantes. Et lorsque le vulgarisateur bute sur un mot, il s'agit en fait d'un hellénisme: trapezita (en 9, 3, p. 89 ; cf. p. 53 et n. 220). Mais la réécriture n'est à l'évidence pas neutre : trois exemples très bien choisis suffisent à démontrer comment le traducteur recherche avant tout la concision. Par souci de simplification, il supprime les métaphores qui alourdissent l'expression en en obscurcissant le sens; et à l'inverse, par souci d'explicitation, il n'hésite pas à ajouter des paraphrases ou à restituer les mots sous-entendus, lorsqu'il ne va pas jusqu'à gloser le texte de Cassien. Le but de ce volgarizzamento est bel et bien didactique : il faut rendre le texte absolument limpide, et même facilement assimilable. Prenons un seul exemple, l'explicit de la Conlatio, qui réunit à lui seul les divers aspects étudiés dans l'introduction et montre que la traduction fidèle du texte latin ne se fait pas sans un léger infléchissement. Le latin « Talibus uerbis nos beatus Moyses erudiens » est ainsi rendu : "Quisti sunu li paroli e precetti per li quali simu stati, supra la virtuti di la discretioni, amaistrati da l'abbati Moyses» (p. 64-65).

5 Mais, aussi riches que soient les remarques relatives à l'intérêt linguistique de l'œuvre, elles ne sont pas l'objet principal de l'introduction: la physionomie de la langue du Raxunamentu donnera lieu à un examen systématique dans une publication ultérieure. Cette première approche se donne surtout pour tâche de tenter de reconstruire le cadre socio-culturel qui a vu apparaître cette réécriture. Et la question, posée à propos de l'ensemble des volgarizzamenti siciliens, du type de lecteurs envisagé par le « traducteur ", trouve une réponse très convaincante s'agissant du Raxunamentu : comme l'ajout de precetti et d'amaistrati dans l'explicit le laissait déjà entrevoir, les destinataires pourraient être les moines eux-mêmes, ou plutôt - puisque ces derniers sont censés maîtriser la langue latine - les convers ou les moins alphabétisés. Mais pourquoi choisir comme vecteur le sicilien, qu'une partie seulement des moines connaît, et alors qu'on peut facilement disposer de textes en langue toscane? L'hypothèse qu'avance l'éditeur est hardie, mais très séduisante: ce choix pourrait s'expliquer par la sensibilité 
religieuse du "traducteur ", qui chercherait à valoriser le rôle de la volonté humaine contre la doctrine de la toute-puissance exclusive de la grâce, en donnant aux moines les moins instruits, grâce à un texte en vulgaire, le moyen de se former eux-mêmes. Et cette formation commencerait par la vertu de discrétion, qui sied bien à l'esprit des congrégations observantes.

6 C'est dans une Nota al testo, curieusement placée après l'édition, qu'on lira toutes les remarques concernant le manuscrit $S$, les critères de l'édition et surtout la recherche $\mathrm{du}$ modèle latin, ou du moins de la famille de laquelle on pourrait rapprocher ce dernier. Une confrontation du texte du Raxunamentu avec l'apparat critique de la seule édition dont on dispose des Collationes (celle de M. Petschenig dans le CSEL 13, 1886), puis avec d'autres témoins italiens, mais surtout avec l'édition bâloise d'Amerbach de 1497, permet à l'éditeur d'identifier le texte dont s'est servi le vulgarisateur anonyme et d'expliquer les nombreux problèmes de contamination. Il s'agit d'un exemplaire de l'édition Amerbach, annoté par un moine de Sant'Eugenio di Siena et aujourd'hui conservé à Palerme; et cette découverte, qui apporte à l'éditeur une solide base, confère à l'ensemble des remarques de détail qui émaillent l'introduction une autorité très forte.

7 L'édition proprement dite présente les caractéristiques qui sont habituelles dans le cas d'un unicum : le texte du manuscrit y est fidèlement retranscrit, les folios mentionnés et les abréviations résolues (à cet égard, l'emploi de l'italique est peut-être superflu). En bas de la page figurent deux apparats distincts: un apparat critique indiquant les leçons du manuscrit, soit qu'elles ont été corrigées ensuite (par le copiste lui-même [cf. p. 109-112]), soit, très rarement, qu'il faut rejeter comme évidemment fausses (dans le cas de lettres mal formées ou de syllabes bissées) ; un second apparat donne quelquesunes des variantes des témoins manuscrits ou imprimés. Sur toutes ces questions de présentation du texte, l'éditeur s'explique en détail dans sa Nota. On aurait aimé multiplier les fines remarques que fait F. Raffaele dans l'introduction, en confrontant directement l'original et sa traduction (mise en vis-à-vis?). Mais on peut bien comprendre le choix de l'éditeur. On devra donc, pour mener à bien ce travail, disposer de l'édition $d u \mathrm{Xv}^{\mathrm{e}}$ siècle (dont on trouvera, par exemple, un exemplaire numérisé à cette adresse : http://tudigit.ulb.tu-darmstadt.de/show/inc-ii-486, mais qui ne contient évidemment pas les annotations de l'incunable de Palerme).

On trouvera enfin, précédant une bibliographie exemplaire, un glossaire de tous les mots siciliens. Si la lemmatisation en rend la consultation très commode, il ne faudra pas manquer de préférer, pour des recherches multiples et sans doute plus fécondes, l'outil offert par le Corpus Artesia, disponible en ligne (http://artesia.ovi.cnr.it), qui permettra à terme d'interroger également l'ensemble des textes de la littérature en vulgaire sicilien. Précisons toutefois que, pour le Raxunamentu, seules sont valables pour le moment les références de l'édition imprimée : l'édition électronique n'ayant pas été mise à jour, et comme la numérotation des phrases a entretemps été modifiée, les résultats obtenus sont quelquefois différents pour le début du texte, mais le sont systématiquement à partir de la phrase 3 du chapitre 9. Ce glossaire sera, sans aucun doute, très précieux pour l'étude de l'évolution de la langue sicilienne, dont de nombreux mots trouvent dans le Raxunamentu leur toute première occurrence. Peutêtre les latinistes pourraient-ils, là encore, regretter que ce lexique soit uniquement un lexique sicilien-italien et que le latin de Cassien n'y figure que très occasionnellement. 
9 Mais on comprend bien que le but de cette édition, et de la collection en général, est avant tout de fournir un matériau neuf, indispensable pour toute étude, ce qui est déjà suffisamment ambitieux. Concernant plus particulièrement Cassien et ses Collationes, cette nouvelle édition non seulement fournit une preuve supplémentaire de l'importance de la diffusion et de la réception médiévales d'une des œuvres fondatrices du monachisme occidental, mais manifeste surtout l'évidente nécessité d'une étude générale de cette tradition. 